allele and genotype frequencies polymorphisms were obtained by direct counting. In each group the Hardy-Weinberg equilibrium was evaluated using the ${ }^{2}$ test. Associations were assessed using odds ratio (OR). Stata v.12.0 program was used to analyse data. The construction and analysis of haplotypes was performed using Haploview v.4.2

Results: In total 70 patients were HLA-B27 positive and 34 were HLA-B15 positive. 78 were women and 100 were men. Linkage disequilibrium map of the ERAP gene is depicted in figure 1. When analysed by ERAP2 haplotype it is observed that there is a statistically significant association with the combinations described in table 1. No associations were observed between ERAP1 haplotypes and HLA-B15 or B27

Conclusions: In the group of patients analysed, a statistically significant association was found between patients with SpA HLA-B15 positive and the haplotype TGT of ERAP2. Also HLA-B27 positive SpA patients were associated with haplotype TGC and CAT of ERAP2 with statistical significance

Disclosure of Interest: None declared

DOI: 10.1136/annrheumdis-2017-eular.5659

\section{THU0006 TRANS-ETHNIC META-ANALYSIS OF GENOME-WIDE ASSOCIATION STUDIES IDENTIFIES GSDMA AND PRDM1 AS SUSCEPTIBILITY GENES TO SYSTEMIC SCLEROSIS}

C. Terao $^{1}$, T. Kawaguchi ${ }^{2}$, P. Dieude ${ }^{3}$, J. Varga ${ }^{4}$, M. Kuwana ${ }^{5}$, M. Hudson ${ }^{6}$, Y. Kawaguchi ${ }^{7}$, M. Matucci-Cerinic ${ }^{8}$, K. Ohmura ${ }^{2}$, G. Riemekasten ${ }^{9}$, A. Kawasaki ${ }^{10}$, P. Airo ${ }^{11}$, T. Horita ${ }^{12}$, A. Oka ${ }^{13}$, E. Hachulla ${ }^{14}$, H. Yoshifuiji ${ }^{2}$, P. Caramaschi ${ }^{15}$, N. Hunzelmann ${ }^{16}$, M. Baron ${ }^{6}$, T. Atsumi ${ }^{12}$, P. Hassouns ${ }^{17}$ A. Tochimoto ${ }^{18}$, N. Ayuzawa ${ }^{19}$, H. Yanagida ${ }^{19}$, H. Furukawa ${ }^{10}, \mathrm{~S}$. Tohma ${ }^{20}$ M. Hasegawa ${ }^{21}$, M. Fujimoto ${ }^{22}$, O. Ishikawa ${ }^{23}$, T. Yamamoto ${ }^{24}$, D. Goto ${ }^{10}$, Y. Asano ${ }^{25}$, M. Jinnin ${ }^{26}$, H. Endo ${ }^{27}$, H. Takahashi ${ }^{28}$, K. Takehara ${ }^{29}$, S. Sato ${ }^{25}$, H. $\operatorname{lhn}^{26}$, S. Raychaudhuri ${ }^{1}$, K. Liao ${ }^{1}$, P. Gregersen ${ }^{30}$, N. Tsuchiya ${ }^{10}$, V. Riccieri ${ }^{31}$, I. Melchers ${ }^{32}$, G. Valentini ${ }^{33}$, A. Cauvet ${ }^{34}$, M. Martinez ${ }^{35}$, T. Mimori ${ }^{2}$, F. Matsuda ${ }^{2}$, Y. Allanore ${ }^{36}$. ' ${ }^{1}$ Brigham and Women's Hospital, Boston, United States; ${ }^{2}$ Kyoto University Graduate School of Medicine, Kyoto, Japan; ${ }^{3}$ Rheumatology Bichat Hospital, Paris, France; ${ }^{4}$ Northwestern University Feinberg School of Medicine, Chicago, United States; ${ }^{5}$ Keio University School of Medicine, Tokyo, Japan; ${ }^{6}$ Jewish General Hospital and Lady Davis Research Institute, Montreal, Canada; ${ }^{7}$ Institute of Rheumatology, Tokyo Women's Medical University, Tokyo, Japan; ${ }^{8}$ University of Florence, Firenze, Italy; ${ }^{9}$ University of Lübeck, Lübeck, Germany; ${ }^{10}$ University of Tsukuba, Tsukuba, Japan; ${ }^{11}$ Spedali Civili, Brescia, Italy; ${ }^{12}$ Hokkaido University Graduate School of Medicine, Sapporo; ${ }^{13}$ Tokai University, Isehara, Japan; ${ }^{14}$ Lille University, Lille, France; ${ }^{15}$ Azienda Ospedaliera Universitaria Integrata, Verona, Italy; ${ }^{16}$ University of Koln, Koln, Germany; ${ }^{17}$ Johns Hopkins University, Baltimore, United States; ${ }^{18}$ Tokyo Women's Medical University, Tokyo; ${ }^{19}$ Utano National Hospital, Kyoto; ${ }^{20}$ Sagamihara Hospital, National Hospital Organization, Sagamihara;

${ }^{21}$ University of Fukui, Fukui; ${ }^{22}$ Tsukuba, University of Tsukuba; ${ }^{23}$ Gunma University Graduate School of Medicine, Gunma; ${ }^{24}$ Fukushima Medical University, Fukushima; ${ }^{25}$ University of Tokyo Graduate School of Medicine, Tokyo; ${ }^{26}$ Kumamoto University, Kumamoto; ${ }^{27}$ Toho University, Tokyo; ${ }^{28}$ Sapporo Medical University School of Medicine, Sapporo; ${ }^{29}$ Kanazawa University, Kanazawa, Japan; ${ }^{30}$ The Feinstein Institute for Medical Research, Manhasset, United States; ${ }^{31}$ Sapienza University of Rome, Rome, Italy; ${ }^{2}$ University Medical Center, Freiburg, Germany; ${ }^{33}$ Second University of Naples, Naples, Italy; ${ }^{34}$ Paris Descartes University, Paris; ${ }^{35}$ Batiment B Purpan Hospital, Toulouse; ${ }^{36}$ Paris Descartes University, Paris, France

Background: Systemic sclerosis (SSc) is an autoimmune disease characterized by fibrosis and composed of two subtypes, limited and diffuse cutaneous forms. Previous genetic studies including genome-wide association studies (GWAS) have identified 12 susceptibility loci satisfying genome-wide significance.

Objectives: To expand the list of susceptibility genes and deepen biological insights for SSc.

Methods: We performed trans-ethnic meta-analysis of GWAS in the Japanese and European populations, followed by a two-staged replication study comprising a total of 4,436 cases and 14,751 controls. Associations between significant single nuclear polymorphisms (SNPs) and neighboring genes were evaluated. Enrichment analysis of $\mathrm{H} 3 \mathrm{~K} 4 \mathrm{Me} 3$, a representative histone mark for active promoter was conducted with an expanded list of SSc susceptibility genes.

Results: We identified two significant SNP in two loci, GSDMA and PRDM1, both of which are related with immune functions and associated with other autoimmune diseases $\left(\mathrm{p}=1.4 \times 10^{-10}\right.$ and $6.6 \times 10^{-10}$, respectively). GSDMA also showed a significant association with limited cutaneous SSc. We also replicated the associations of previously reported loci including a non-GWAS locus, TNFAIP3. PRDM1 encodes BLIMP1, a transcription factor regulating T cell proliferation and plasma cell differentiation. The top SNP in GSDMA was a missense variant and correlated with gene expression of neighboring genes, and this could explain the association in this locus. We found different HLA association patterns between the two populations or two subtypes. Enrichment analysis suggested the importance of CD4 naïve primary T cell.

Conclusions: GSDMA and PRDM1 are associated with SSc. These findings provide enhanced insight into the genetic and biological basis of SSc.

Disclosure of Interest: None declared

DOI: 10.1136/annrheumdis-2017-eular.2165

\section{THU0007 DEEP SEQUENCING TRANSCRIPTOME ANALYSIS OF THE EFFECT OF TRAUMEEL VERSUS DICLOFENAC THERAPEUTIC ACTION IN WOUND HEALING}

G.St. Laurent III ${ }^{1}$, B. Seilheimer ${ }^{2}$, M. Tackett ${ }^{1}$, J. Zhou ${ }^{1}$, D. Shtokalo ${ }^{1}$, Y. Vyatkin ${ }^{1}$, P. Kapranov ${ }^{1}$, I. Toma ${ }^{3}$, T. Mccaffrey ${ }^{3} .{ }^{1}$ The St. Laurent Institute, Vancouver, WA, United States; ${ }^{2}$ Biologische Heilmittel Heel, GmbH, Baden-Baden, Germany; ${ }^{3}$ Department of Medicine/Division of Genomic Medicine, The George Washington University, Washington, DC, United States

Background: Anti-inflammatory agents are used widely in treating numerous inflammatory conditions. The effect of $\operatorname{Tr} 14$, a multitargeted natural product, was compared to diclofenac, a non-selective cyclooxygenase inhibitor, on cutaneous wound repair in mice.

Objectives: To compare the effect of diclofenac with $\operatorname{Tr} 14$ on the transcriptome after cutaneous wounding in the mouse.

Methods: After abrasive wounding, the wounds were treated with topical $\operatorname{Tr} 14$ or diclofenac at clinically relevant doses. An additional group received subcutaneous Tr14 injections. The healing wounds were analyzed for RNA transcript profiling by RNAseq at specific times (12h, 24h, 36h, 72h, 96h, 120h, 196h) after injury. Differentially expressed genes (DEGs) were computed at each time point between diclofenac vs control or $\operatorname{Tr} 14$ vs control using EdgeR.

Results: Across time points, Tr14 treatment modulated a number of transcripts related to key wound repair pathways such as cellular differentiation, wound contraction, and cell mobility. Diclofenac, in contrast, changed gene expression mainly in two areas: Prominent effects were observed with regard to DNA chromatin regulation and ribosomal function, further effects were observed on the prostaglandin pathway and wound repair factors. In many of the key pathways modulated by Tr14, such as the defense response and cell motility, diclofenac tended to have an opposite effect on gene expression. At 12 hours post-injury, there were 521 transcripts significantly elevated and 1027 transcripts that were decreased by diclofenac treatment. By comparison, using a similar number of transcripts altered by $\operatorname{Tr} 14$ treatment, only 4 transcripts were increased in common, and 5 transcripts were decreased in common, suggesting that the therapies have different effects on the transcriptome.

Conclusions: The overall patterns of the $\operatorname{Tr} 14$ and diclofenac responses in the transcriptome during wound repair are very different. The Tr14 effect is most pronounced on the defense response, cell motility, and anti-apoptotic pathways. In contrast, diclofenac mainly affected histones and chromatin remodeling systems, as well as ribosomal systems that would be expected to alter the translational pattern of diclofenac-treated cells.

Disclosure of Interest: G. St. Laurent, III: None declared, B. Seilheimer: None declared, M. Tackett: None declared, J. Zhou: None declared, D. Shtokalo: None declared, Y. Vyatkin: None declared, P. Kapranov: None declared, I. Toma: None declared, T. Mccaffrey Speakers bureau: TM has received speaker's honorarium from HEEL, GmbH

DOI: 10.1136/annrheumdis-2017-eular.4964

\section{THU0008 MAST CELLS SHOW A REPROGRAMMED TRANSCRIPTIONAL SIGNATURE FOLLOWING REPEATED IGG STIMULATIONS}

T. Messemaker, J. Suurmond, K. Habets, M. Heijink, J. Schonkeren, A. Dorjee, M. Giera, T. Huizinga, R. Toes, F. Kurreeman. LUMC, Leiden, Netherlands

Background: Mast cell numbers are increased in the rheumatoid arthritis joint. We have previously shown that mast cells can be activated by IgG-ACPA leading to the production of proinflammatory cytokines. However, not much is known about the resulting function when mast cells would repeatedly engage IgG, a likely scenario given the long life span of mast cells (up to a year) and the perpetual presence of IgG-ACPA in the joints. We have recently shown that mast cells triggered repeatedly through their Ig Fc epsilon receptor undergo a reprogramming of their responses (Suurmond et al. JACl, 2016 by expressing de-novo transcribed genes in the antigen presentation and pathogen defence response pathways.

Objectives: The aim of the current work was to determine whether mast cells show similar changes in their response mode following repeated interactions with $\lg$.

Methods: Human cord blood-derived mast cells were treated for 2 weeks with plate-bound lgG. The expression profile of naive or treated mast cells was measured through RNA sequencing, quantitative RT-PCR, flow cytometry. Protein secretion was measured with ELISA and Luminex assays. Metabolic changes were measured using HPLC mass-spectrometry.

Results: Similar to our previous work on Fc Epsilon receptor, we observe a dampening of the normal $\operatorname{lgG}$ responses with a set of novel genes upregulated. Interestingly, de-novo expressed genes consisted of DHCR7 and DHCR24, key enzymes in the cholesterol pathway. Pathway analysis confirms an enrichment of genes in this pathway following repeated IgG triggering. Preliminary data on metabolic profiling reveals a decrease in phospholipid levels in repeatedly activated mast cells.

Conclusions: Our study provides evidence that mast cells are reprogrammed upon repeated IgG triggering. In contrast to repeated Fc Epsilon Receptor triggering, different pathways are affected, implying stimulus-specific effects. Our work has important implications for the understanding the role of mast cells in rheumatoid arthritis. 
Disclosure of Interest: None declared

DOI: 10.1136/annrheumdis-2017-eular.6326

\section{THU0009 IN VITRO STUDIES USING CYBRIDS, SHOW THAT MTDNA FROM HEALTHY AND OA PATIENTS HAVE DIFFERENT MITOCHONDRIAL ACTIVITY}

M. Fernández-Moreno ${ }^{1,2}$, A. Dalmao-Fernandez ${ }^{2}$, T. Hermida-Gomez ${ }^{2}$, M.J. Sanchez-Dopico ${ }^{2}$, M.E. Vazquez-Mosquera ${ }^{2}$, E. Cortes-Pereira ${ }^{2}$, S. Relaño-Fernandez ${ }^{2}$, I. Rego-Perez ${ }^{2}$, F.J. Blanco ${ }^{2} .{ }^{1}$ Centro de Investigación Biomédica en Red, Ciber-Bbm, Madrid; ' 2 Servicio de Reumatología. Complexo Hospitalario Universitario (CHUAC), Instituto de Investigación Biomédica de A Coruña (INIBIC), A Coruña, Spain

Background: Mitochondrial dysfunction is well documented in OA and has the capacity to promote abnormalities in chondrocyte function and viability contributing to cartilage degeneration. Previous studies have showed the mtDNA haplogroup $\mathrm{J}$ are associated with incidence and progression of $\mathrm{OA}$. Cybrids are optimal cellular models to study the real mitochondrial biology and function implications in the cellular behaviour, since they carry different mitochondrial variants with the same nuclear background, therefore, excluding the variations because of nuclear genome.

Objectives: The aim of this work is to test the real role of mtDNA in cellular activity, using cybrids with mtDNA from healthy donors (without $O A$ ) and from patients with knee OA.

Methods: Cybrids were developed using 143B.TK- Rho-0 cell line (nuclear donor) and platelets (mitochondrial donors) from healthy (without $\mathrm{AO}-\mathrm{N}-$ ) and knee OA donors. The OXPHOS function was evaluated by SeaHorse XFp after addition of oligomycin, FCCP and Rotenone/Antimycin A. The metabolic status was evaluated by glucose consumption and lactic acid production. The glycolytic activity was measure after addition of glucose, oligomycin and 2-dioxyglucose using Seahorse XFp. Appropriate statistical analyses were performed with GraphPad Prism v6.

Results: OA cybrids had lower basal respiration $(92.07 \pm 39.9$ and $155.5 \pm 54.08$, $p<0.0005)$, and maximal respiratory capacity $(114.7 \pm 50.1$ and $160.6 \pm 44.7$, $\mathrm{p}<0.05$ ) than $\mathrm{N}$. The analysis of ATP production was lower in OA than in N cybrids $(66.69 \pm 28.69$ versus $101.5 \pm 42.04 \mathrm{p} \leq 0.05)$. The $\%$ spare respiratory capacity value for the $\mathrm{N}$ was significantly lower than in $\mathrm{OA}$ cybrids $(107 \pm 16.21$ versus 124.7 $\pm 5.97, p<0.0005)$. Cybrids carrying the mtDNA from OA patients showed higher glucose consumption than $\mathrm{N}$ cybrids $(43.77 \pm 8.87 \mathrm{mg} / \mathrm{ml}$ and $31.91 \pm 13.69$ $\mathrm{mg} / \mathrm{ml} ; \mathrm{p}<0.05$ ) however in the lactic acid production did not exit differences. The glycolytic activity was evaluated and showed that OA cybrids had lower glycolysis $(71.05 \pm 4.83$ versus $85.43 \pm 11.18, \mathrm{p}<0.05)$ but higher glycolytic reserve than $\mathrm{N}$ cybrids $(56.60 \pm 7.5$ versus $39.73 \pm 19.13, p \leq 0.05)$.

Conclusions: Cybrids have different metabolic behaviour, being $\mathrm{N}$ more efficient using glucose via glycolysis. We found differences statistical significate in the parameters that describe the mitochondrial respiration capacity, in this line OA cybrids had lower mitochondrial respiration and produce less ATP than the cybrids obtained from healthy patients. These results showed that the mitochondria obtained from healthy and OA donors had a different behaviour. These data also offer a real rationale for why mitochondria alterations play an important role in the incidence of OA.

Disclosure of Interest: None declared

DOI: 10.1136/annrheumdis-2017-eular.5930

\section{THU0010 ALTERED MIRNAS PROFILES IN PLASMA-DERIVED EXOSOME OF PATIENTS WITH ANKYLOSING SPONDYLITIS BY SMALL RNA-SEQ ANALYSIS}

Y. Huang, T. Li, Z. Huang, W. Deng, S. Zheng, X. Guo, Z. Huang. Guangdong No. 2 Provincial People's Hospital, Guangzhou, China

Background: Ankylosing spondylitis (AS) is a chronic inflammatory disease, which is difficult to diagnose in the early stages. Increasing evidences have shown that MicroRNAs (miRNAs) may serve as novel biomarkers for AS. Exosome can function as vehicles to deliver miRNAs in body fluids including saliva and plasma. However, the relationship between exosome-delivered miRNAs and AS has yet to be determined.

Objectives: The aim of this study is to detect the altered miRNAs profiles of plasma-derived exosome in AS patients by small RNA-Seq Analysis.

Methods: Ribo ${ }^{\mathrm{TM}}$ kit was used to isolate exosome. Small RNA Sample Pre Kit was used to build libraries in 3 AS patients and 3 healthy volunteers (HV), following by IlluminaHiSeq platform sequencing and bioinformatics analysis. Quantitative reverse-transcription PCR (qRT-PCR) was used to confirm the expression of the highly-expressed miRNA in another $10 \mathrm{AS}$ patients and $10 \mathrm{HV}$, and receiveroperator characteristic $(\mathrm{ROC})$ curve was used to evaluate the diagnostic value of miRNAs.

Results: Small RNA-Seq analysis showed that the Q30 value of HV and AS patients were higher than 95\% (Fig.1-A). The amount of miRNA in HV and AS patients were (509.667 \pm 77.501$)$ and (632.000 \pm 43.555$) .80$ up-regulated and 19 down-regulated exosomal miRNAs were identified in AS patients, compared with HV ( $\mid \log 2$ Ratio $>1, P<0.05$ ) (Fig.1-B-C). The target genes of the 34 highly-expressed miRNAs from the 99 differently-expressed miRNAs were 7869 , and the main function of these target genes are involved in the regulation of endocytosis and protein modification process analyzed by GO and KEGG. The qRT-PCR results indicated that the expression level of miRNA21-5P and miRNA423-5P in AS patients were $(2.940 \pm 1.572)$ and $(2.520 \pm 1.401)$ times higher than that of HV (Fig.1-D-E). ROC curve analysis showed that miRNA21-5P and miRNA423-5P had significant diagnostic value for AS with the AUC of 0.890 (CI95\%: 0.723-1.057) and 0.835 (CI95\%: 0.621-1.039) respectively (Fig.1-F).
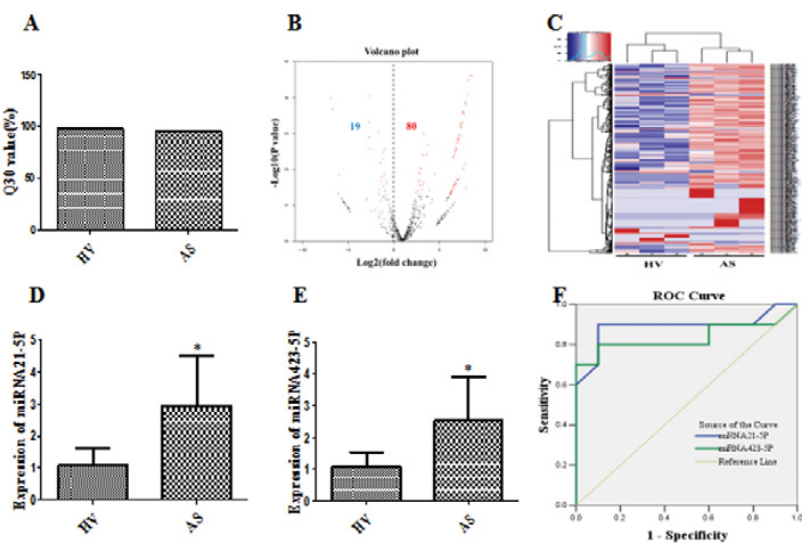

Fig.l Altered miRNAs profiles in plasma-derived exosome of AS by small RNA-Seq Analysis A: The Q30 value of each group. B-C: The differentially-expressed miRNAs. D-E: gRT-PCR was used to confirm the level of miRNA21-5P and miRNA423-5P.F: ROC cure was used to evaluate the tiagnostic value of miRNA21-5P and miRNA423-5P. $P<0.05$ vs HV.

Conclusions: The miRNAs profiles in plasma-derived exosome of AS patients are significant different from HV. miRNA21-5P and miRNA423-5P are higher expressed in AS patients. Thus, plasma-derived exosomal miRNAs might be reliable biomarkers to identify AS.

References:

[1] EI MA. Extra-articular manifestations of ankylosing spondylitis: prevalence, characteristics and therapeutic implications. Eur J Intern Med, 2011, 22(6):554-60.

Disclosure of Interest: None declared

DOI: 10.1136/annrheumdis-2017-eular.5637

\section{THU0011 IMMUNE SIGNAL 2 CHECKPOINT MOLECULE EXPRESSION IN RHEUMATOID ARTHRITIS DISEASE PROGRESSION}

A.M. Walsh ${ }^{1}$, M. Canavan ${ }^{2}$, Y. Guo ${ }^{1}$, T. McGarry ${ }^{2}$, X. Yin ${ }^{1}$, M.D. Wechalekar ${ }^{3}$, M.D. Smith ${ }^{3}$, S.M. Proudman ${ }^{4}$, C. Orr ${ }^{5}$, S. Kelly ${ }^{6}$, C. Pitzalis ${ }^{6}$, D.J. Veale ${ }^{5}$, U. Fearon ${ }^{2}$, S. Nagpal ${ }^{1}$. ' Janssen R\&d, Spring House, United States;

${ }^{2}$ Molecular Rheumatology, Trinity Biomedical Sciences Institute, Trinity College Dublin, Dublin, Ireland; ${ }^{3}$ Flinders University; ${ }^{4}$ Royal Adelaide Hospital and Discipline of Medicine, University of Adelaide, Adelaide, Australia; ${ }^{5}$ St. Vincent's University Hospital, Dublin, Ireland; ${ }^{6}$ Queen Mary University of London, London, United Kingdom

Background: Deep profiling of synovial tissue samples from rheumatoid arthritis (RA) patients may reveal the molecular underpinnings of phases of RA progression and provide new therapeutic targets to intervene earlier in disease pathogenesis. Objectives: We sought to identify the molecular pathways expressed in different stages of disease (from seropositive subjects without clinically apparent synovitis to those with established disease) in synovial tissue compared to non-RA controls. Methods: Transcriptomics profiling was performed on RNA isolated from synovial tissue biopsies. Normal synovium was collected from subjects with knee pain and without diagnosis of OA or RA $(n=28)$. Arthralgia tissue was collected from ACPA-positive subjects without synovitis $(n=10)$. Early RA tissue was collected from patients recently diagnosed ( $<1$ year) with $R A(n=57)$. Established RA tissue was collected from ACPA-positive subjects with $>1$ year of disease duration $(n=95)$. Protein expression was confirmed on infiltrating immune cells from synovial biopsy cell suspensions by flow cytometry in separate RA subjects.

Results: Several pathways previously identified as important for RA pathogenesis (e.g., lymphocyte activation, osteoclast differentiation, NF-kappa B signaling) were enriched in differentially expressed genes in disease synovial biopsies compared to normal tissue samples. Interestingly, several genes known to function in $T$ cell activation as signal 2 co-stimulatory or co-inhibitory molecules were differentially expressed, even in arthralgia and early RA subjects. 66 of 81 known co-stimulatory or co-inhibitory genes profiled were differentially expressed (FDR $<5 \%$ and absolute fold-change $>2$ ) in disease samples from at least one cohort. The genes encoding co-stimulatory proteins that were increased compared to normal included CD28, CD4OLG, CD40 and ICOS. Interestingly, some of the genes encoding co-inhibitory proteins were increased (PDCD1/PD-1, CD274/PDL1, HAVCR2/TIM3, TIGIT, BTLA), whereas others showed decreased expression (C10orf54/VISTA and LAG3) compared to normal controls. We focused on CD28 expression, which is elevated in "pre-RA" arthralgia samples, proposing that anti-CD28 therapeutics could be candidates for RA disease prevention. By flow cytometry we demonstrated that a majority of CD4+ (>90\%) and CD8+ $(>60 \%) \mathrm{T}$ 